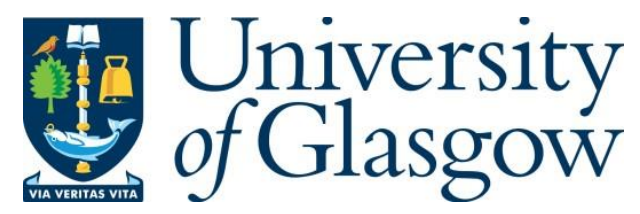

Zhang, X., Abbasi, Q. H., Fioranelli, F., Romain, O. and Le Kernec, J. (2022) Elderly Care - Human Activity Recognition Using Radar with an Open Dataset and Hybrid Maps. In: 16th EAI International Conference on Body Area Networks (EAI

BODYNETS 2021), Glasgow, UK, 25-26 Oct 2021, pp. 39-51. ISBN 9783030955922.

There may be differences between this version and the published version. You are advised to consult the publisher's version if you wish to cite from it.

https://eprints.gla.ac.uk/250637/

Deposited on: 31 August 2021

Enlighten - Research publications by members of the University of Glasgow https://eprints.gla.ac.uk 


\title{
Elderly Care - Human Activity Recognition using Radar with an Open Dataset and Hybrid Maps
}

\author{
Xinyu Zhang ${ }^{1,2}$, Qammer H. Abbasi ${ }^{10000-0002-7097-9969]}$, Francesco Fioranelli3 ${ }^{[0000-0001-}$ \\ 8254-8093], Olivier Romain ${ }^{40000-0002-2172-1865]}$ and Julien Le Kernec ${ }^{1,2[0000-0003-2124-6803]}$ \\ ${ }^{1}$ University of Glasgow, University Avenue, G12 8QQ, Glasgow, UK. \\ ${ }^{2}$ University of Electronic Science and Technology of China, Qingshuihe Campus: Chengdu \\ High-tech Zone (West) 2006 West Avenue, source Zip: 611731, China. \\ ${ }^{3}$ TU Delft, Mekelweg 4, 2628 CD Delft, The Netherlands. \\ ${ }^{4}$ University Cergy-Pontoise, 6 av du ponceau, 95000 Cergy-Pontoise, France. \\ julien.lekernec@glasgow.ac.uk
}

\begin{abstract}
Population ageing has become a severe problem worldwide. Human activity recognition (HAR) can play an important role to provide the elders with in-time healthcare. With the advantages of environmental insensitivity, contactless sensing and privacy protection, radar has been widely used for human activity detection. The micro-Doppler signatures (spectrograms) contain much information about human motion and are often applied in HAR. However, spectrograms only interpret magnitude information, resulting in suboptimal performances. We propose a radar-based HAR system using deep learning techniques. The data applied came from the open dataset "Radar signatures of human activities" created by the University of Glasgow. A new type of hybrid map was proposed, which concatenated the spectrograms amplitude and phase. After cropping the hybrid maps to focus on useful information, a convolutional neural network $(\mathrm{CNN})$ based on LeNet-5 was designed for feature extraction and classification. In addition, the idea of transfer learning was applied for radar-based HAR to evaluate the classification performance of a pre-trained network. For this, GoogLeNet was taken and trained on the newly-produced hybrid maps. These initial results showed that the LeNet-5 CNN using only the spectrograms obtained an accuracy of $80.5 \%$, while using the hybrid maps reached an accuracy of $84.3 \%$, increasing by $3.8 \%$. The classification result of transfer learning using GoogLeNet was $86.0 \%$.
\end{abstract}

Keywords: Human activity recognition, convolutional neural network, transfer learning, radar, micro-Doppler, hybrid maps.

\section{Introduction}

\subsection{Context}

The development of public health and disease control has contributed much to increasing human life expectancy. However, along with the decreased average fertility 
rates, human longevity has resulted in the global issue of population ageing. World Population Ageing Report 2019 by the United Nations [1] summarised the previous trend of the population distribution in the world for the past two decades and estimated the trend for the next 30 years, from 2019 to 2050 . The result showed the proportion of the global population for the old (aged over 65) had experienced a continuous increase, from around $6 \%$ in 1990 to $9 \%$ in 2019. Following the growing trend, the projected ageing population in 2050 would account for nearly $16 \%$.

Human Activity Recognition (HAR) aims at conducting classification tasks to accurately identify human activities for further promoting proactive and timely healthcare [2]. Up to now, the most common methods of human activity detection are visionbased detection like using cameras and sensor-based detection such as using wearable sensors, radar and smartphone sensors [3,4]. Among all the methods, radar technology outperforms the other for the following aspects [5-8]. Environmental insensitivity: radar detection is not influenced by harsh light; Contactless Sensing: users do not need to wear or connect with any devices, which provides a high capability of comfort and convenience; Privacy Protection: radar technology collects human activity data without showing their actual images, ensuring the privacy of individuals.

Much of the research [3,9-11] around radar-based human activity recognition revolves around micro-Doppler signatures (spectrograms) [12], which provide rich information for classification.

\subsection{Current Research Progress}

Feature Extraction.

In the research on HAR, it is apparent that one essential part is to extract features from the spectrograms and identify the movements represented in them. It can be found in previous research works that the features commonly used can be divided into several categories:

\section{Physical Characteristics}

Physical characteristics are characteristics with physical meanings. For instance, Kim [13] selected six features from a micro-Doppler map. Six features were extracted from the spectrogram, including the torso frequency where the scattering is strongest, the total bandwidth, the overall frequency shift, the Doppler bandwidth without micro-Dopplers, the normalised standard deviation (STD) of the signal intensity and the activity period. These are then used for classification. Further examples can be found for healthcare and animal welfare applications with physical characteristic extraction [3-5,14-21].

\section{Micro-Doppler Maps (Spectrograms).}

One main inconvenience of the feature extraction method based on extracting handcrafted features is that it relies heavily on the know-how of the radar engineer. As machine learning developed, many researchers began to directly consider the grayscale of RGB images of spectrograms as features. Then, convolutional neural 
networks derived from vision-based classification were applied to those images for classification $[3-7,10,22]$. Compared with the conventional hand-crafted feature extraction approaches, the use of deep learning technology can increase the accuracy in classification. Through training and testing of a large number of data samples, deep learning seeks out the mapping relationship between data features and labels [23].

\section{Classification Approaches.}

There have been numerous research works in solving HAR tasks. Because the movements of the human body are complex and each micro-motion can produce a unique micro-Doppler signature, many researchers have delved into extracting specific features from the micro-Doppler spectrograms and using classifiers to perform specific classification tasks.

At the start, conventional machine learning techniques, such as random forest, support vector machine (SVM) and K-nearest neighbour classifiers, were commonly researched. In 2009, Kim's team firstly used a support vector machine to identify activities from human micro-Doppler features. [13] In the experiment, the volunteers were asked to perform seven different activities. Then, they designed a variety of empirical features for each movement and extracted parameters according to those features. Finally, the team classified those seven activities by training a support vector machine model and received an average classification accuracy of $91.4 \%$. In [24], a random forest was performed to classify the data information in a real-time manner. As a result, the average gesture recognition precision reached over $90 \%$.

In recent years, with the development of GPU and deep neural networks, they have been applied to the human activity classification based on micro-Doppler characteristics as a powerful classifier. Deep learning technology can implement feature extraction and activity classification simultaneously, with minimal input from the human operator. In [25], the authors detected the micro-motion information of drones and obtained the micro-Doppler maps. They then carried out frequency-domain transformation to the Doppler features and obtained the cadence-velocity diagram (CVD). The two feature maps were merged and classified by the deep convolutional neural network. As a result, the accuracy reached more than $90 \%$ in drone classification. Kim [26] directly applied a deep convolutional neural network to the raw radar microDoppler spectrograms for classifying seven human activities. An accuracy of $90.9 \%$ was achieved.

Compared with traditional machine learning techniques, neural networks can independently extract features from the input data and reach higher classification accuracy. Deep learning techniques do not depend on the prior knowledge of the input data and tend to obtain better classification performance in more complex situations, at the price of increased need of labelled data amount for proper training.

At present, most of the research on radar-based HAR utilise the micro-Doppler map for feature extraction and activity classification. However, for some activities with similar movements, one map can be easily identified as the other map, producing the wrong prediction. Therefore, research attention on alternative or complementary radar data representations such as hybrid $2 \mathrm{D}$ maps to reduce the rate of false alarms increases. In [27], the authors incorporate the three-domain maps, time-Doppler map 
(micro-Doppler), time-range map and range-Doppler map. Three stacked autoencoders were used to extract features and then fed the features into three softmax classifiers. The results showed that the classification accuracy gained after combining the three classifiers reached $96 \%$, increasing from $93.3 \%$ using the micro-Doppler map, $95.4 \%$ using the range map and $90 \%$ using the range-Doppler map. From the study, it can be observed that though the micro-Doppler spectrograms contain rich information of the raw radar data, the possibility of false alarms is a challenge in radar-based HAR. Therefore, it is necessary to combine more information with the micro-Doppler maps to improve the HAR system performance.

For a raw radar echo received, the data contain I and Q channels, which can be synthesised into a complex signal. After the radar signal processing, the result is still in the complex form $[8,28]$, through which the magnitude and phase information can both be extracted. However, the majority of the researches on radar-based HAR only investigate the effect of the magnitude information but ignore the effect of the phase information. In [29], the authors use Histogram Oriented Gradient features from range maps exploiting both the phase information showed superior classification performance compared to amplitude range maps.

Hence, the question arises on whether processing the phase of the data can bring any benefit for classification. In order to increase the activity classification performance and reduce confusion between activities, we propose a novel approach by jointly processing the amplitude and the phase information of micro-Doppler patterns for recognition.

The remainder of this article is organised as follows. Section 2 - Methodology and Implementation - introduces the implementation of the HAR system in detail. With the hybrid maps created after radar signal processing, the implementation procedures of the self-designed CNN and GoogLeNet are explained in detail. Section 3 - Results and Discussion - describes the hardware and software environments for the experiment and presented the classification results in three situations. Then, it discusses the classification performances, the results of the classification will be presented and analysed. Finally, Section 5 - Conclusions and Future Work - summarises the contents and major contributions. Then, based on the discussion section, it identifies possible directions for further research.

\section{$2 \quad$ Methodology and Implementation}

\subsection{Dataset information}

The data used in this paper come from an open dataset recorded by the University of Glasgow, "Radar signatures of human activities" [30]. The team used an FMCW radar, SDR-KIT-580B, produced by Ancortek with waveform generation and transmission modules, transmitting and receiving antennas, RF cables and other accessories, as shown in Fig. 1. The radar was operated at C-band $(5.8 \mathrm{GHz})$, and the bandwidth was set as $400 \mathrm{MHz}$ [22]. During the experiment, ten activities were performed by volunteers, as shown in Table 1 . This is one of the largest dataset in radarbased HAR. The full details of the experiment are described in $[22,30]$ 


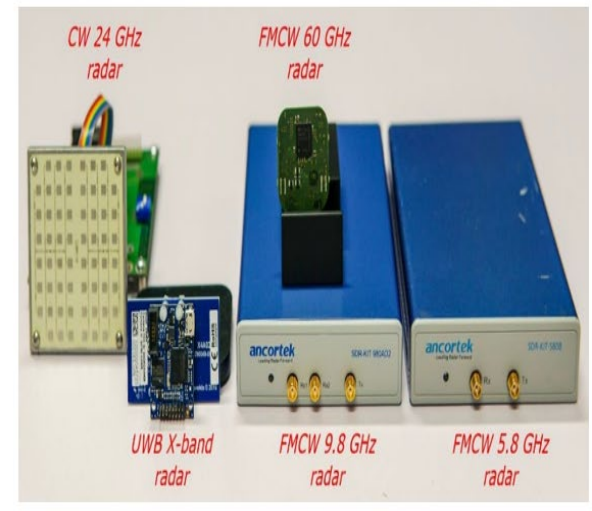

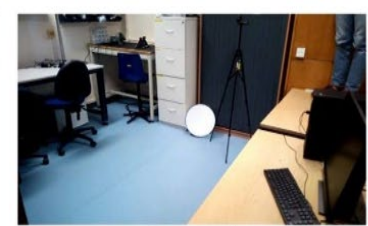

Room A

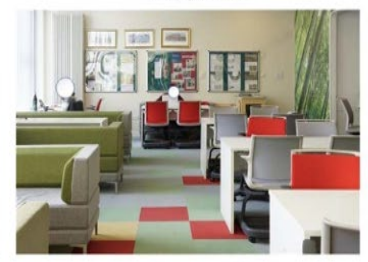

Room C

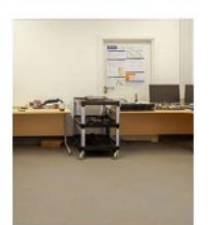

Room B

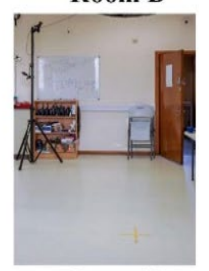

Room D

Fig. 1. The left shows the radar systems used by the radar team at the University of Glasgow. They used the rightmost radar, SDR-KIT-580B of Ancortek, to create the open dataset. The right shows four examples of the experiment environments for data collection adapted from $[3,22]$.

Table 1. 10 Activities recorded in the open dataset with their according duration. Note that the class "Not interested" includes four different tasks that the software should classify as not of interest for the classification to emulate an open dataset containing a multitude of classes and the detection of the classes of interest from all the activities that could be performed in front of the radar.

\begin{tabular}{|c|c|c|}
\hline $\begin{array}{c}\text { Number of } \\
\text { samples }\end{array}$ & Activity Name & Duration [s] \\
\hline 250 & Walking & 10 \\
\hline 316 & Sitting on a chair & 5 \\
\hline 313 & Standing up from a chair & 5 \\
\hline 314 & Bending down and pick up a pen & 5 \\
\hline 314 & Drinking couple of sips & 5 \\
\hline 226 & Falling down & 5 \\
\hline \multirow{2}{*}{284} & Not interested $\left\{\begin{array}{c}\text { Checking under the } \\
\text { bed }\end{array}\right.$ & 10 \\
\hline $\begin{array}{c}\text { Moving objects } \\
\text { Answering a phone } \\
\text { call }\end{array}$ & 10 \\
\hline \multirow{2}{*}{$\begin{array}{c}\text { Bending and tying } \\
\text { shoelaces }\end{array}$} & 5 \\
\hline
\end{tabular}




\subsection{Pre-processing}

The chirps have a pulse repetition period of $1 \mathrm{~ms}$ with 128 samples per sweep. A fast Fourier transform is applied on the collected I\&Q signal to extract the range information for each sweep (fast time) to obtain a range profile. A moving target indicator is used to suppress static targets from the radar returns. After collecting enough range profiles, a short-time Fourier transform is applied over each range bin (slow time). The window size is set to $200 \mathrm{~ms}$, the overlap factor to $95 \%$ for a smooth spectrogram, and the zero-padding factor to 4 for a finer Doppler resolution. After this pre-processing, the spectrogram amplitude and phase can be extracted, as shown in Fig. 2. Before extracting features from micro-Doppler patterns, it is necessary to concatenate the phase information with the amplitude information. Also, from the microDoppler signature shown below. To further improve the performance, the spectrogram is cropped to contain the actions with the maximum Doppler and discarding the Doppler bins at higher and lower frequencies not containing the target signature. The resulting concatenated image is $120 \times 340 \times 3$. The $120 \times 340$ corresponds to the number of pixels per channel, and there are 3 channels for Red, Green and Blue.

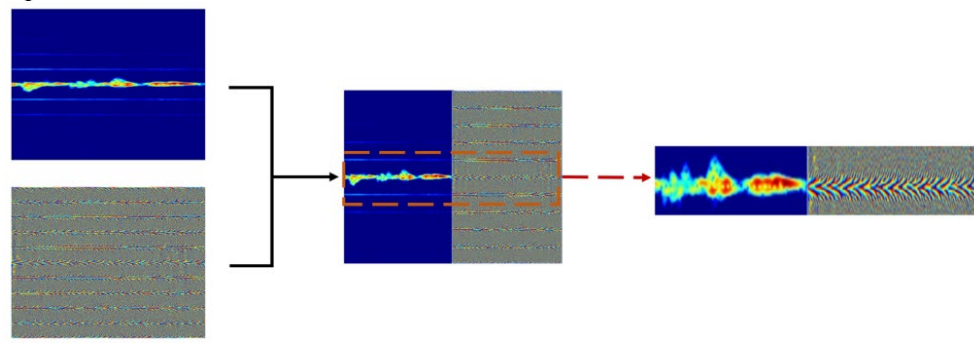

Fig. 2. The processing chain of micro-Doppler signatures and phase plots. Firstly, combine the two plots into one. Then, extract the main information inside the red dashed box by cropping the resulting image.

The hybrid maps of the 10 activities are shown in Fig. 3. Some activities can be easily distinguished, like moving objects and falling, while others are more difficult to tell apart because of the similarity of the micro-Doppler amplitude patterns, like answering a phone and picking up a pen. In total, there were 2017 images used in this project, and the number of samples per class is shown in Table 1. 


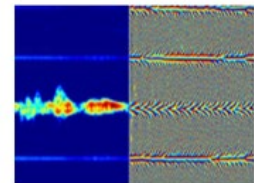

(a) Answering a phone call

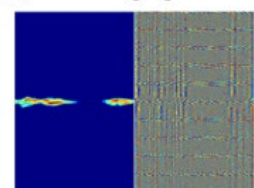

(e) Drinking couple of sips

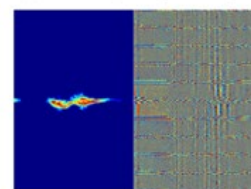

(i) Standing up from a chair

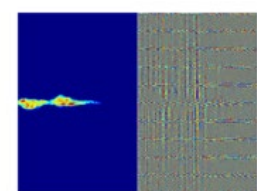

(b) Picking up a pen

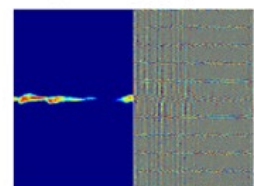

(f) Falling down

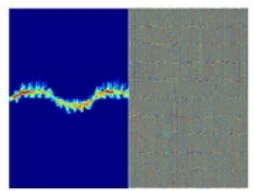

(j) Walking

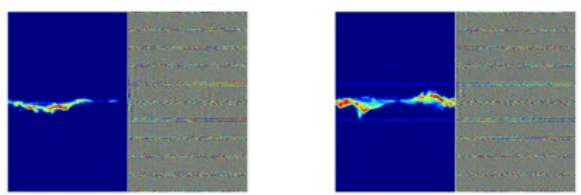

(c) Bending down and tying shoelaces (d) Checking under the bed

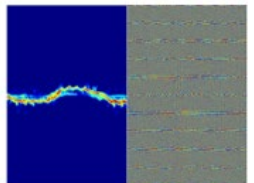

(g) Moving objects

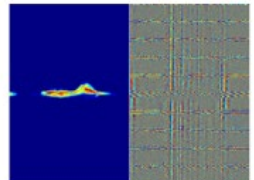

(h) Sitting on a chair

Fig. 3. Hybrid maps of the ten activities recorded in the open dataset, which concatenate micro-Doppler amplitude and phase.

\subsection{Feature extraction and classification}

The processed data inputs are in the form of RGB images. Thus, convolutional networks $(\mathrm{CNN})$ normally used for image recognition were selected to extract features automatically and classify the data.

\section{CNN Based on LeNet-5}

Derived from [31], the key parameters of the convolutional and pooling layers are summarised in Table 2, where all units are pixels, for the proposed CNN based on LeNet5. The dropout layer was put to prevent data overfitting during the training process. After each convolutional layer, batch normalisation is implemented to normalise the output produced. The purpose of this layer is to speed up the convergence of model performance. The drop rate was set to be 0.5 to avoid overfitting. In this implementation, ReLU was used as the activation function for all nodes except in the output layer, which uses Softmax. 


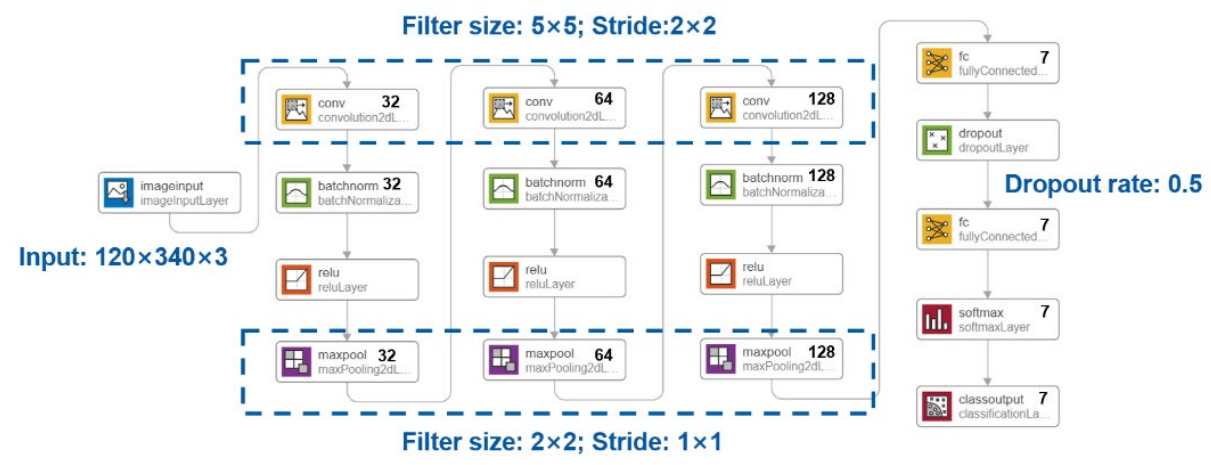

Fig. 4. Layout of the LeNet5-based CNN

Table 2. Parameters of the self-designed $\mathrm{CNN}$ about convolutional and pooling layers

\begin{tabular}{|c|c|c|c|c|c|c|}
\hline $\begin{array}{c}\text { Convolutional } \\
\text { Layer }\end{array}$ & $\begin{array}{c}\text { Filter } \\
\text { Number }\end{array}$ & $\begin{array}{c}\text { Filter } \\
\text { Size }\end{array}$ & Stride & $\begin{array}{c}\text { Pooling } \\
\text { Layer }\end{array}$ & $\begin{array}{c}\text { Pooling } \\
\text { Area }\end{array}$ & Stride \\
\hline Conv_1 & 32 & $5 \times 5$ & $1 \times 1$ & maxpool_1 & $2 \times 2$ & $1 \times 1$ \\
\hline Conv_2 & 64 & $5 \times 5$ & $1 \times 1$ & maxpool_2 & $2 \times 2$ & $1 \times 1$ \\
\hline Conv_3 & 128 & $5 \times 5$ & $1 \times 1$ & maxpool_3 & $2 \times 2$ & $1 \times 1$ \\
\hline
\end{tabular}

\section{Transfer Learning - Use Pre-trained Model GoogLeNet}

Transfer learning utilises a pre-trained model of one task to accomplish another task. The main reasons for applying transfer learning are as follows. Pre-trained models like VGG [32], GoogLeNet [33] and ResNet [34] have been tested on large datasets and performances have been optimised. The second reason is that sometimes there is insufficient data to generalise from, so using a pre-trained model can prevent data overfitting [35]. In this study, transfer learning with GoogLeNet is also tested.

The data samples were divided into three sets, where a fixed $10 \%$ of data composed the test set. For the remaining dataset, because the dataset is not large and to prevent overfitting, five-fold cross-validation was applied. Hence, $80 \%$ of the remaining data were set as the training set, while the other $20 \%$ were the validation set.

\section{$3 \quad$ Results and Discussion}

\subsection{Hardware and Software Environment}

The radar signal processing, feature extraction and image classification were all implemented using Matlab. With the help of a GPU, the networks were developed and trained using the Deep Learning Toolbox and Parallel Computing Toolbox in MATLAB. The hardware and software environments are introduced in Table 3. 
Table 3. Hardware parameters and software versions

$\begin{array}{ll}\text { CPU } & \text { Intel(R) Core (TM) i7-8700 3.2GHz } \\ \text { RAM Capacity } & 8192 \mathrm{MB} \\ \text { MATLAB } & 2021 \mathrm{a} \\ \text { Graphics Processing Unit (GPU) } & \text { NVIDIA GeForce RTX } 3070 \\ \text { GPU Total Memory } & 8.59 \mathrm{~GB}\end{array}$

\subsection{Classification Results}

Firstly, the HAR system was tested with training the CNN based on LeNet5.As for the hyperparameters, the initial learning rate for training was set as 0.0001 , and the maximum epoch number was 20 , as this was enough for convergence. There were 16 data samples in each mini-batch, and the training and validation data would be shuffled for every epoch. This guaranteed complete training with all data inputs. In terms of the validation process, one validation operation was performed every 20 iterations. The stochastic gradient descent with momentum (SGDM) optimiser was chosen to update the weights.

To evaluate the improvement over amplitude only classification, spectrograms (amplitude only size $120 \times 340 \times 3)$ and the new hybrid images $(120 \times 340 \times 3)$ were both trained using the CNN based on LeNet5. The confusion matrix for amplitude only is shown in Fig. 5 top left and for the hybrid maps in Fig.5 top right.

For Transfer learning, the hybrid maps were resized to $224 \times 224 \times 3$. In addition, the last layers of GoogLeNet were replaced by new layers designed to fit the classification task. The confusion matrix is shown in Fig.5, bottom left. A comparison of the overall performances of the 3 methods is shown in Fig. 5 bottom right.

\subsection{Discussion}

From Fig. 5, note that the hybrid maps which concatenate the magnitude and phase information produce higher accuracy than the spectrograms (amplitude only), with the classification accuracy increasing by $3.8 \%$. The pre-trained GoogLeNet performed $1.7 \%$ better than the CNN based on LeNet5 at the cost of increased computational requirements. From the three confusion matrices, it is clear that compared with other activities, the activities labelled "Not interested" and "Picking up a pen" are confused for one another. After concatenating the spectrograms with phase plots, the average classification accuracy improved in these two categories by $20.9 \%$ and $0 \%$, respectively, for the CNN based on LeNet 5, and $15.6 \%$ and $15.1 \%$ for the GoogLeNet implementation. Thus, the proposed hybrid patterns can enhance the radar-based HAR performance.

Also, it is worth noting that through the training progress of GoogLeNet, though the mini-batch training accuracy converged to near $100 \%$ as the training went by, the validation accuracy was smaller than $100 \%$ (86 89\% in the five folds), and so did the 
test accuracy. This may be caused by data overfitting. One possible reason is that the depth of GoogLeNet in MATLAB is 22, and the parameters are around 7 million [36] as opposed to $60 \mathrm{k}$ for LeNet5.
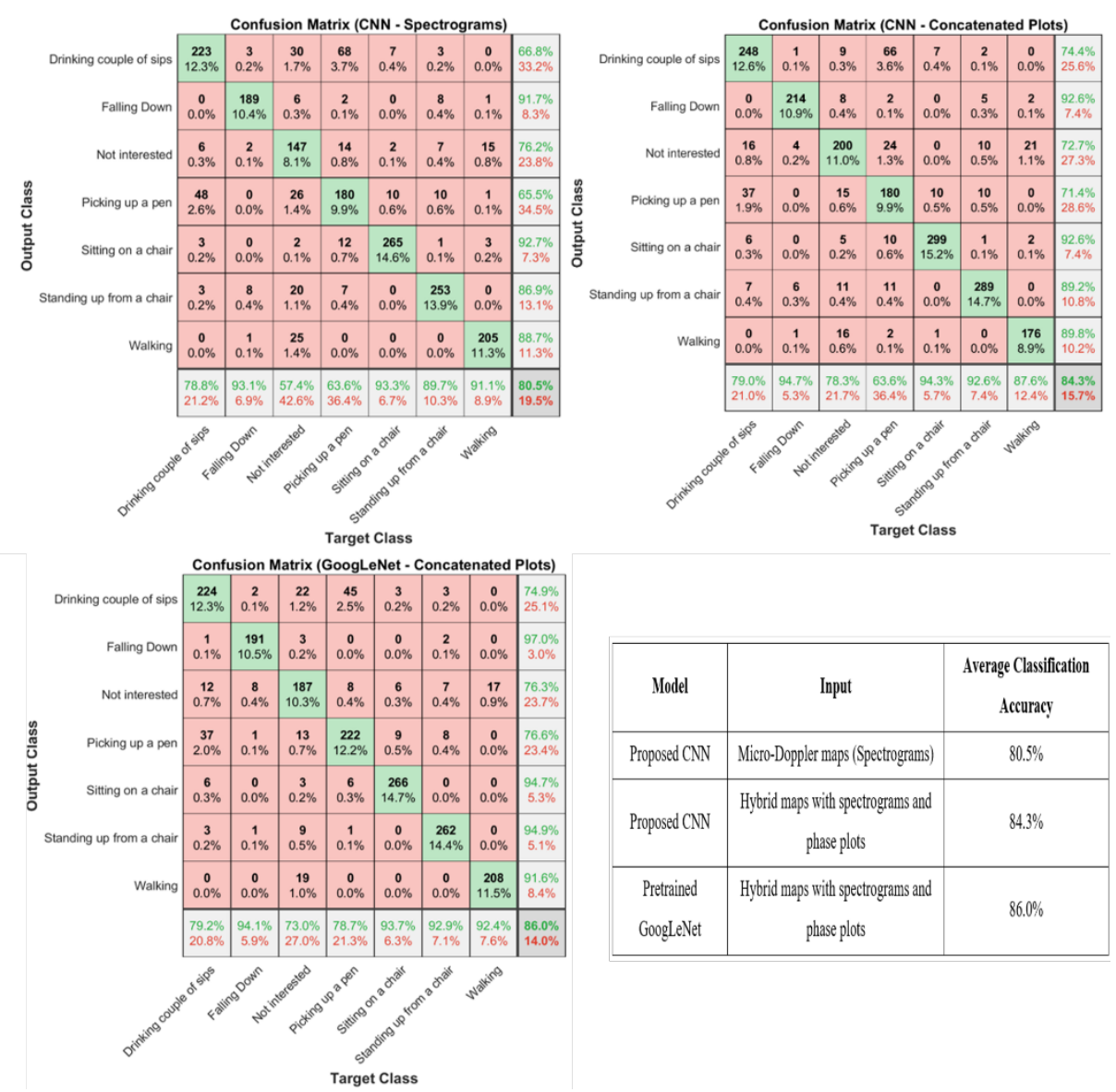

\begin{tabular}{|c|c|c|}
\hline Model & Input & $\begin{array}{c}\text { Average Classification } \\
\text { Accuracy }\end{array}$ \\
\hline Proposed CNN & Micro-Doppler maps (Spectrograms) & $80.5 \%$ \\
\hline Proposed CNN & $\begin{array}{c}\text { Hybrid maps with spectrograms and } \\
\text { phase plots }\end{array}$ & $84.3 \%$ \\
\hline $\begin{array}{l}\text { Pretrained } \\
\text { GoogLeNet }\end{array}$ & $\begin{array}{c}\text { Hybrid maps with spectrograms and } \\
\text { phase plots }\end{array}$ & $86.0 \%$ \\
\hline
\end{tabular}

Fig. 5. Confusion matrix obtained using, top left) the self-designed $\mathrm{CNN}$ on spectrograms, top right) the self-designed $\mathrm{CNN}$ on hybrid maps, bottom left) the pre-trained GoogLeNet, bottom right) comparison of different methods for radar-based HAR.

\section{Conclusions and Future Work}

In this study, the micro-Doppler amplitude and phase were combined to produce hybrid maps of radar signatures. After cropping the images onto the Doppler bins containing target signature, the proposed CNN based on LeNet5 improved the classification accuracy by $3.8 \%$ compared to amplitude only and 5.5\% with GoogLeNet via transfer learning. These results show that exploiting the complex nature of the signals 
is essential in improving performances, as suggested in [37] calling to use complex numbers in machine learning architectures

Future directions for this project would look at data augmentation to increase the size of the dataset for better model generalisation. Popular augmentation methods include image rotation, reflection and scaling along the $\mathrm{x}$ or $\mathrm{y}$ axis, cropping and translation $[38,39]$. However, in radar, not all those techniques are valid as radar images have physical meaning. For example, rotation is not valid as this would never be produced by a radar system.

The raw radar data is typically processed to obtain information in three domains: range, time and velocity (can be described by Doppler shift) [3-5]. The spectrograms and phase plots were combined as a hybrid map. Range-time and range-Doppler maps could also be used to create hybrid maps containing amplitude and phase information either by concatenation or by adding the phase information as supplemental channels in CNN. These could be combined with deep fusion to further improve performance $[40,41]$.

\section{References}

1. D. o. E. a. S. A. United Nations, Population Division (2020), "World Population Ageing 2019."

2. A. Bulling, U. Blanke, and B. Schiele, "A tutorial on human activity recognition using body-worn inertial sensors," ACM Comput. Surv., vol. 46, no. 3, p. Article 33, 2014, doi: $10.1145 / 2499621$.

3. J. L. Kernec et al., "Radar Signal Processing for Sensing in Assisted Living: The challenges associated with real-time implementation of emerging algorithms," IEEE Signal Processing Magazine, vol. 36, no. 4, pp. 29-41, 2019, doi: 10.1109/MSP.2019.2903715.

4. Imran, M.A., Ghannam, R., Abbasi, Q.H., Fioranelli, F. and Kernec, J.L. (2021). Contactless Radar Sensing for Health Monitoring. In Engineering and Technology for Healthcare (eds M.A. Imran, R. Ghannam and Q.H. Abbasi). https://doi.org/10.1002/9781119644316.ch2

5. F. Fioranelli, J. L. Kernec, and S. A. Shah, "Radar for Health Care: Recognizing Human Activities and Monitoring Vital Signs," IEEE Potentials, vol. 38, no. 4, pp. 16-23, 2019, doi: 10.1109/MPOT.2019.2906977.

6. M. Jia, S. Li, J. L. Kernec, S. Yang, F. Fioranelli and O. Romain, "Human activity classification with radar signal processing and machine learning," 2020 International Conference on UK-China Emerging Technologies (UCET), 2020, pp. 1-5, doi: 10.1109/UCET51115.2020.9205461.

7. S. Li, M. Jia, J. L. Kernec, S. Yang, F. Fioranelli and O. Romain, "Elderly Care: Using Deep Learning for Multi-Domain Activity Classification," 2020 International Conference on UK-China Emerging Technologies (UCET), 2020, pp. 1-4, doi: 10.1109/UCET51115.2020.9205464.

8. S. Yang, J. L. Kernec, F. Fioranelli and O. Romain, "Human Activities Classification in a Complex Space Using Raw Radar Data," 2019 International Radar Conference (RADAR), 2019, pp. 1-4, doi: 10.1109/RADAR41533.2019.171367.

9. X. Li, Y. He, and X. Jing, "A Survey of Deep Learning-Based Human Activity Recognition in Radar," Remote. Sens., vol. 11, p. 1068, 2019. 
10. S. Z. Gurbuz and M. G. Amin, "Radar-Based Human-Motion Recognition With Deep Learning: Promising applications for indoor monitoring," IEEE Signal Processing Magazine, vol. 36, no. 4, pp. 16-28, 2019, doi: 10.1109/MSP.2018.2890128.

11. J. L. Kernec, F. Fioranelli, S. Yang, J. Lorandel and O. Romain, "Radar for assisted living in the context of Internet of Things for Health and beyond," 2018 IFIP/IEEE International Conference on Very Large Scale Integration (VLSI-SoC), 2018, pp. 163-167, doi: 10.1109/VLSI-SoC.2018.8644816.

12. V. C. Chen, The Micro-Doppler Effect in Radar. Artech House Publishers, 2011.

13. Y. Kim and H. Ling, "Human Activity Classification Based on Micro-Doppler Signatures Using a Support Vector Machine," IEEE Transactions on Geoscience and Remote Sensing, vol. 47, no. 5, pp. 1328-1337, 2009, doi: 10.1109/TGRS.2009.2012849.

14. F. Fioranelli et al., "Radar-based evaluation of lameness detection in ruminants: preliminary results," 2019 IEEE MTT-S International Microwave Biomedical Conference (IMBioC), 2019, pp. 1-4, doi: 10.1109/IMBIOC.2019.8777830.

15. K. Linardopoulou, L. Viora, Q.H. Abbasi, F. Fioranelli, J. Le Kernec, and N. Jonsson (2020) Lameness Detection in Dairy Cows: Evaluation of the Agreement and Repeatability of Mobility Scoring. 74th Annual AVTRW Conference, 14-15 Sept 2020.

16. V. Busin, L. Viora, G. King, M. Tomlinson, J. Le Kernec, N. Jonsson, and F. Fioranelli, (2019) Radar Sensing as a Novel Tool to Detect Lameness in Sheep. Ontario Small Ruminant Veterinary Conference, Ontario, Canada, 17-19 June 2019.

17. A. Shrestha, C. Loukas, J. Le Kernec, F. Fioranelli, V. Busin, N. Jonsson, G. King, M. Tomlinson, L. Viora, and L. Voute (2018) Animal lameness detection with radar sensing. IEEE Geoscience and Remote Sensing Letters, 15(8), pp. 1189-1193. (doi: 10.1109/LGRS.2018.2832650)

18. V. Busin, L. Viora, G. King, M. Tomlinson, J. Le Kernec, N. Jonsson and F. Fioranelli, (2019) Evaluation of lameness detection using radar sensing in ruminants. Veterinary Record, 185(18), 572. (doi: 10.1136/vr.105407) (PMID:31554712)

19. A. Shrestha, J. Le Kernec, F. Fioranelli, J.F. Marshall and L. Voute (2018) Gait Analysis of Horses for Lameness Detection with Radar Sensors. In: RADAR 2017: International Conference on Radar Systems, Belfast, UK, 23-26 Oct 2017, ISBN 9781785616730 (doi:10.1049/cp.2017.0427)

20. H. Li, A. Shrestha, F. Fioranelli, J. Le Kernec, H. Heidari, M. Pepa, E. Cippitelli, E. Gambi and S. Spinsante (2017) Multisensor Data Fusion for Human Activities Classification and Fall Detection. In: IEEE Sensors 2017, Glasgow, UK, 30 Oct - 01 Nov 2017, ISBN 9781509010127 (doi:10.1109/ICSENS.2017.8234179)

21. X. Li, Z. Li, F. Fioranelli, S. Yang, O. Romain, and J. L. Kernec, "Hierarchical Radar Data Analysis for Activity and Personnel Recognition," Remote Sensing, vol. 12, no. 14, p. 2237, Jul. 2020..

22. Fioranelli, F., Shah, S. A., Li, H., Shrestha, A., Yang, S. and Le Kernec, J. (2019) Radar sensing for healthcare. Electronics Letters, 55(19), pp. 1022-1024. (doi: 10.1049/el.2019.2378)

23. Y. LeCun, Y. Bengio, and G. Hinton, "Deep learning," Nature, vol. 521, no. 7553, pp. 436444, 2015/05/01 2015, doi: 10.1038/nature14539.

24. K. A. Smith, C. Csech, D. Murdoch, and G. Shaker, "Gesture Recognition Using mmWave Sensor for Human-Car Interface," IEEE Sensors Letters, vol. 2, no. 2, pp. 1-4, 2018 , doi: 10.1109/LSENS.2018.2810093.

25. B. K. Kim, H. Kang, and S. Park, "Drone Classification Using Convolutional Neural Networks With Merged Doppler Images," IEEE Geoscience and Remote Sensing Letters, vol. 14, no. 1, pp. 38-42, 2017, doi: 10.1109/LGRS.2016.2624820. 
26. Y. Kim and T. Moon, "Human Detection and Activity Classification Based on MicroDoppler Signatures Using Deep Convolutional Neural Networks," IEEE Geoscience and Remote Sensing Letters, vol. 13, no. 1, pp. 8-12, 2016, doi: 10.1109/LGRS.2015.2491329.

27. B. Jokanovic, M. Amin, and B. Erol, "Multiple joint-variable domains recognition of human motion," in 2017 IEEE Radar Conference (RadarConf), 8-12 May 2017 2017, pp. 0948-0952, doi: 10.1109/RADAR.2017.7944340.

28. C. Loukas, F. Fioranelli, J. Le Kernec and S. Yang, "Activity Classification Using Raw Range and I \& Q Radar Data with Long Short Term Memory Layers," 2018 IEEE 16th Intl Conf on Dependable, Autonomic and Secure Computing, 16th Intl Conf on Pervasive Intelligence and Computing, 4th Intl Conf on Big Data Intelligence and Computing and Cyber Science and Technology Congress(DASC/PiCom/DataCom/CyberSciTech), 2018, pp. 441-445, doi: 10.1109/DASC/PiCom/DataCom/CyberSciTec.2018.00088.

29. R. G. Guendel, F. Fioranelli and A. Yarovoy, "Phase-Based Classification for Arm Gesture and Gross-Motor Activities Using Histogram of Oriented Gradients," in IEEE Sensors Journal, vol. 21, no. 6, pp. 7918-7927, 15 March15, 2021, doi: 10.1109/JSEN.2020.3044675.

30. F. Fioranelli, Shah, S. A. , Li, H., Shrestha, A., Yang, S. and Le Kernec, J. Radar signatures of human activities, doi: 10.5525/gla.researchdata.848.

31. Y. Lecun, L. Bottou, Y. Bengio, and P. Haffner, "Gradient-based learning applied to document recognition," Proceedings of the IEEE, vol. 86, no. 11, pp. 2278-2324, 1998, doi: $10.1109 / 5.726791$.

32. C. Szegedy, W. Liu, Y. Jia, P. Sermanet, S. Reed, D. Anguelov, D. Erhan, V. Vanhoucke, and A. Rabinovich, "Going deeper with convolutions," Proc. CVPR, 2015, arXiv: 1409.4842

33. K. Simonyan and A. Zisserman, "Very deep convolutional networks for large-scale image recognition,", Proc. CVPR, 2014, arXiv:1409.1556.

34. K. He, X. Zhang, S. Ren, and J. Sun, "Deep residual learning for image recognition,” Proc. CVPR, 2016, arXiv:1512.03385.

35. E. S. Olivas, J. D. M. Guerrero, M. M. Sober, J. R. M. Benedito, and A. J. S. Lopez, Handbook Of Research On Machine Learning Applications and Trends: Algorithms, Methods and Techniques - 2 Volumes. Information Science Reference - Imprint of: IGI Publishing, 2009.

36. MATLAB. "Pretrained Deep Neural Networks." The MathWorks Inc. https://www.mathworks.com/help/deeplearning/ug/pretrained-convolutional-neuralnetworks.html (accessed April 19, 2021).

37. J. Bruna, S. Chintala, Y. LeCun, S. Piantino, A. Szlam, M. Tygert, “A mathematical motivation for complex-valued convolutional networks", 2015, arXiv:1503.03438v3

38. A. Gandhi. "Data Augmentation | How to use Deep Learning when you have Limited Data-Part 2." Nanonets. https://nanonets.com/blog/data-augmentation-how-to-use-deeplearning-when-you-have-limited-data-part-2/ (accessed April, 19, 2021).

39. J. Li, A. Shrestha, J. Le Kernec and F. Fioranelli (2019) From Kinect skeleton data to hand gesture recognition with radar. Journal of Engineering, 2019(20), pp. 6914-6919. (doi: 10.1049/joe.2019.0557)

40. H. Li, J. le Kernec, A. Mehul, S. Z. Gurbuz and F. Fioranelli, "Distributed Radar Information Fusion for Gait Recognition and Fall Detection," 2020 IEEE Radar Conference (RadarConf20), 2020, pp. 1-6, doi: 10.1109/RadarConf2043947.2020.9266319.

41. H. Li, A. Mehul, J. Le Kernec, S. Z. Gurbuz and F. Fioranelli, "Sequential Human Gait Classification With Distributed Radar Sensor Fusion," in IEEE Sensors Journal, vol. 21, no. 6, pp. 7590-7603, 15 March15, 2021, doi: 10.1109/JSEN.2020.3046991. 